\title{
Laparoscopic repair of inguinal hernias: Risk factors for urinary retention and chronic pain after totally extraperitoneal repair and transabdominal preperitoneal repair
}

\author{
Sung Gu Kim, Jungtack Son, Sung Ryol Lee, Kyung Uk Jung \\ Department of Surgery, Kangbuk Samsung Hospital, Sungkyunkwan University School of Medicine, Seoul, Korea
}

Purpose: There are various opinions about the postoperative complications of the two methods for laparoscopic inguinal hernia surgery; totally extraperitoneal repair (TEP) and transabdominal preperitoneal repair (TAPP). The aim of this study was to compare the postoperative course after TAPP and TEP, focusing on immediate postoperative pain, incidence of postoperative urinary retention (POUR), and chronic pain.

Methods: This study retrospectively analyzed a consecutive series of 344 inguinal hernia patients who were treated with laparoscopic surgery between November 2016 and December 2019 at a single tertiary referral center.

Results: Patient demographics did not differ significantly between the groups. The operation time was significantly shorter in the TEP group than in the TAPP group ( $43.1 \pm 14.9$ minutes vs. $63.5 \pm 16.5$ minutes, $p<0.001)$. The postoperative pain scores were significantly lower in the TEP group than in the TAPP group immediately (3.6 \pm 1.3 vs. $4.4 \pm 1.1, p<0.001)$ and 6 hours $(1.5 \pm 1.4$ vs. $2.3 \pm 1.8, p<0.001)$ after the operation. The other complications did not differ significantly between the groups. Age was a significant risk factor for POUR (odds ratio [OR], 1.083; 95\% confidence interval [CI], 1.018-1.151; $p=0.011$ ), and history of benign prostate hyperplasia (BPH) was a significant risk factor for chronic pain (OR, 5.363; 95\% CI, $1.028-27.962 ; p=0.046$ ).

Conclusion: TEP and TAPP seem to be safe and effective for laparoscopic inguinal hernia repair and have similar postoperative outcomes. Age was a significant risk factor for POUR, and BPH history was a significant risk factor for chronic pain.
Received September 1, 2021

Revised 1st October 13, 2021 2nd October 29, 2021

Accepted October 30, 2021

Corresponding author

Kyung Uk Jung

Department of Surgery, Kangbuk Samsung Hospital, Sungkyunkwan

University School of Medicine, 29

Saemunan-ro, Jongno-gu, Seoul 03181, Korea

Tel: $+82-2-2001-8408$

Fax: +82-2-2001-8360

E-mail: sahelgrean@gmail.com ORCID:

https://orcid.org/0000-0002-8844-3724

Sung Gu Kim and Jungtack Son contributed equally to this paper as co-first authors.

Keywords: Herniorrhaphy, Totally extraperitoneal, Transabdominal preperitoneal, Chronic pain, Urinary retention

This is an Open Access article distributed under the terms of the Creative Commons Attribution Non-Commercial License (http:// creativecommons.org/licenses/by-nc/4.0/) which permits unrestricted non-commercial use, distribution, and reproduction in any medium, provided the original work is properly cited.

Copyright (c) The Korean Society of Endoscopic and Laparoscopic Surgeons.

\section{INTRODUCTION}

Surgical repair of an inguinal hernia is one of the most common surgical procedures worldwide $[1,2]$. Since the laparoscopic techniques have entered the field of general surgery in the early 1990s, new surgical methods have been introduced for hernia repair. Surgeons have actively adopted minimally invasive techniques for inguinal hernia repair, and the initial cases of two different laparoscopic hernia repair-transabdominal preperitoneal repair (TAPP) and totally extraperitoneal repair (TEP)-were reported in 1992 and 1993, respectively [3,4]. Many clinical trials compared these two representative methods of laparoscopic in- 
guinal hernia repair, whereas conflicting results were produced. Although several randomized controlled trials and meta-analyses have been reported, the clarity of the results has been limited by the small number of patients and heterogeneity of the endpoints $[5,6]$. Although the HerniaSurge Group stated in its guideline for laparoscopic hernia repair published in 2018, "as TAPP and TEP have comparable outcomes it is recommended that the choice of the technique should be based on the surgeon's skills, education, and experience." [7], the data to determine the characteristics of each method is still insufficient.

The aim of this study was to compare the postoperative course after TAPP and TEP by focusing on immediate postoperative pain, incidence of postoperative urinary retention (POUR), and chronic pain.

\section{MATERIALS AND METHODS}

\section{Patients and data collection}

This study retrospectively analyzed a consecutive series of inguinal hernia patients who were treated with laparoscopic surgery between November 2016 and December 2019 at a single tertiary referral center. Patients aged 18 years and older at the time of the surgery were included in the study. Patients with recurrent, incarcerated, or strangulated hernia were excluded from the analysis. Two experienced specialists decided on the operation method according to their respective surgeon's preference.

Patient demographics including age, sex, body mass index (BMI), American Society of Anesthesiologists (ASA) physical status (PS) classification, type of hernia (direct, indirect, femoral, or pantaloon), and location of hernia (right, left, or bilateral)

Table 1. Patients' characteristics

\begin{tabular}{|c|c|c|c|c|}
\hline Characteristic & Total & TEP & TAPP & $p$ value \\
\hline No. of patients & 344 & 226 & 118 & \\
\hline Age (yr) & $62.2 \pm 16.0$ & $62.9 \pm 14.9$ & $60.7 \pm 17.9$ & 0.250 \\
\hline Sex & & & & 0.279 \\
\hline Female & $28(8.1)$ & $21(9.3)$ & $7(5.9)$ & \\
\hline Male & 316 (91.9) & $205(90.7)$ & $111(94.1)$ & \\
\hline BMI $\left(\mathrm{kg} / \mathrm{m}^{2}\right)$ & $23.8 \pm 2.7$ & $23.7 \pm 2.9$ & $23.9 \pm 2.5$ & 0.432 \\
\hline ASA PS classification & & & & 0.399 \\
\hline I & $98(28.5)$ & $59(26.1)$ & $39(33.1)$ & \\
\hline$\|$ & $171(49.7)$ & 118 (52.2) & $53(44.9)$ & \\
\hline III & $73(21.2)$ & $47(20.8)$ & $26(22.0)$ & \\
\hline IV & $2(0.6)$ & $2(0.9)$ & $0(0)$ & \\
\hline Type & & & & 0.098 \\
\hline Direct & $97(28.2)$ & $59(26.1)$ & $38(32.2)$ & \\
\hline Indirect & 231 (67.2) & $159(70.4)$ & $72(61.0)$ & \\
\hline Femoral & $2(0.6)$ & $2(0.9)$ & $0(0)$ & \\
\hline Pantaloon & $14(4.1)$ & $6(2.7)$ & $8(6.9)$ & \\
\hline Location & & & & 0.667 \\
\hline Right & $187(54.4)$ & $126(55.8)$ & $61(51.7)$ & \\
\hline Left & $156(45.4)$ & $99(43.8)$ & $57(48.3)$ & \\
\hline Bilateral & $1(0.3)$ & $1(0.4)$ & $0(0)$ & \\
\hline BPH history & & & & 0.375 \\
\hline No & 291 (84.6) & $194(85.8)$ & $97(82.2)$ & \\
\hline Yes & 53 (15.4) & $32(14.2)$ & $21(17.8)$ & \\
\hline
\end{tabular}

Values are presented as number only, mean \pm standard deviation, or number (\%).

TEP, totally extraperitoneal repair; TAPP, transabdominal preperitoneal repair; BMl, body mass index; ASA, American Society of Anesthesiologists; PS, physical status; BPH, benign prostate hyperplasia. 
were analyzed. A previous history of benign prostate hyperplasia (BPH) was also investigated given its direct association with the risk of POUR.

The surgical outcomes included operation time, rate of conversion to open surgery, degree of postoperative pain, length of postoperative hospital stay, and postoperative complications. Postoperative pain was measured using a numeric rating scale (NRS) immediately; and 6 hours and 24 hours after the operation. To evaluate POUR, the number of postoperative Nelaton catheterizations and whether a Foley catheter was inserted were investigated. In general, the Foley catheter was inserted when the urinary retention was not resolved after two postoperative Nelaton catheterizations. Any events that required additional treatment besides routine clinical procedures, prolonged hospital stay, or frequent outpatient clinic visits were regarded as postoperative complications. Postoperative chronic pain was defined according to previous studies as pain lasting for 6 months after the operation [8-10].

\section{Surgical techniques}

All operations were performed by two attending surgeons with expertise in laparoscopic techniques, and the type of procedure was selected according to the surgeon's preference.

Laparoscopic TEP was performed using a two-port technique.
After creating a channel between the rectus muscle and the posterior sheath at the umbilicus level, a wound retractor and a custom-made glove port comprising a surgical glove were inserted. Dissection was started from a small space between the rectus muscle and the posterior sheath. With $\mathrm{CO}_{2}$ gas insufflation, soft tissue was dissected, and the small vessels were cauterized with electrocautery. During dissection, another 5-mm trocar was inserted into the suprapubic area. After identification of Cooper's ligament and Bogros' space, the hernia sac was assessed and ligated. We used Parietex Anatomical Mesh $(13 \times 9 \mathrm{~cm}$; Covidien, Mansfield, MA, USA) and fixed it with Tacker (Autosuture, Norwalk, CT, USA) and Greenplast-Q (Green Cross Corp., Cheongju, Korea).

Laparoscopic TAPP was performed using a three-port technique. A $10-\mathrm{mm}$ optical trocar was placed in the umbilicus, and two 5-mm operating trocars were inserted bilaterally in the midclavicular line, 1 to $2 \mathrm{~cm}$ below the level of the horizontal line from the umbilicus. After creating the pneumoperitoneum and identifying anatomical landmarks, the peritoneal incision started from the anterior superior iliac spine to the medial umbilical ligament, with a large opening of the preperitoneal space. The rest of the procedure was the same as that used in the TEP technique.

Table 2. Surgical outcomes

\begin{tabular}{|c|c|c|c|c|}
\hline Variable & Total $(n=344)$ & $\operatorname{TEP}(n=226)$ & $\operatorname{TAPP}(n=118)$ & $p$ value \\
\hline Operation time (min) & $50.1 \pm 18.3$ & $43.1 \pm 14.9$ & $63.5 \pm 16.5$ & $<0.001$ \\
\hline Open conversion & $7(3.1)$ & $7(3.1)$ & $0(0)$ & 0.053 \\
\hline \multicolumn{5}{|l|}{ Postoperative pain NRS score } \\
\hline Immediate postoperative & $3.9 \pm 1.3$ & $3.6 \pm 1.3$ & $4.4 \pm 1.1$ & $<0.001$ \\
\hline Postoperative $6 \mathrm{hr}$ & $1.8 \pm 1.6$ & $1.5 \pm 1.4$ & $2.3 \pm 1.8$ & $<0.001$ \\
\hline Postoperative $24 \mathrm{hr}$ & $0.4 \pm 1.0$ & $0.4 \pm 0.9$ & $0.6 \pm 1.2$ & 0.070 \\
\hline Postoperative hospital stay (day) & $1.2 \pm 0.5$ & $1.2 \pm 0.5$ & $1.3 \pm 0.6$ & 0.062 \\
\hline No. of Nelaton catheterizations & $0.2 \pm 0.5$ & $0.2 \pm 0.5$ & $0.2 \pm 0.6$ & 0.294 \\
\hline Foley insertion & $20(5.8)$ & $13(5.8)$ & $7(5.9)$ & 0.921 \\
\hline Postoperative complication & $41(11.9)$ & $27(11.9)$ & $14(11.9)$ & 0.982 \\
\hline Wound seroma & $24(7.0)$ & $14(6.2)$ & $10(8.5)$ & 0.431 \\
\hline Wound infection & $10(2.9)$ & $8(3.5)$ & $2(1.7)$ & 0.504 \\
\hline Wound hematoma & $2(0.6)$ & $1(0.4)$ & $1(0.9)$ & $>0.999$ \\
\hline Chronic pain & $6(1.7)$ & $3(1.3)$ & $3(2.5)$ & 0.417 \\
\hline Mesh removal & $0(0)$ & $0(0)$ & $0(0)$ & $>0.999$ \\
\hline Recurrence & $1(0.3)$ & $1(0.4)$ & $0(0)$ & $>0.999$ \\
\hline
\end{tabular}

Values are presented as mean \pm standard deviation or number (\%).

TEP, totally extraperitoneal repair; TAPP, transabdominal preperitoneal repair; NRS, numeric rating scale. 


\section{Statistical analysis}

Statistical analyses were conducted using IBM SPSS for Windows version 26.0 (IBM Corp., Armonk, NY, USA). Continuous variables were compared using Student $t$ test or the Mann-Whitney $U$ test, and categorical variables were analyzed using the chisquare test or Fisher exact test. The risk values for chronic pain and Foley catheter insertion were calculated using a logistic regression model. The $p$ values $<0.05$ were considered significant.

\section{RESULTS}

\section{Patient demographics}

All 344 patients underwent laparoscopic repair for a primary inguinal hernia during the study period; 226 (65.7\%) were treated with TEP and 118 (34.3\%) with TAPP. The mean age of all patients was $62.2 \pm 16.0$ years. Most of the patients were men (91.9\%, 316 of 344). Patient demographics, including age, sex, BMI, ASA PS classification, type and location of the hernia, and BPH history did not differ significantly between the two surgical groups (Table 1).

Table 3. Risk factor of Foley catheter insertion

\begin{tabular}{|c|c|c|c|c|}
\hline \multirow{2}{*}{ Variable } & \multicolumn{2}{|c|}{ Univariate } & \multicolumn{2}{|c|}{ Multivariate } \\
\hline & $\mathrm{OR}(95 \% \mathrm{Cl})$ & $p$ value & $\mathrm{OR}(95 \% \mathrm{Cl})$ & $p$ value \\
\hline \multicolumn{5}{|l|}{ Operation method } \\
\hline TEP & 1 (Reference) & & & \\
\hline TAPP & $1.043(0.404-2.689)$ & 0.931 & & \\
\hline Age & $1.116(1.055-1.180)$ & $<0.001$ & $1.083(1.018-1.151)$ & 0.011 \\
\hline \multicolumn{5}{|l|}{ Sex } \\
\hline Female & 1 (Reference) & & & \\
\hline Male & $1.733(0.224-13.437)$ & 0.599 & & \\
\hline BMl & $0.864(0.721-1.034)$ & 0.111 & & \\
\hline \multicolumn{5}{|l|}{ Type } \\
\hline Direct & 1 (Reference) & & & \\
\hline Indirect & 2.307 (0.656-8.108) & 0.192 & & \\
\hline Femoral & NA & NA & & \\
\hline Pantaloon & $2.385(0.231-24.666)$ & 0.466 & & \\
\hline \multicolumn{5}{|l|}{ Location } \\
\hline Right & 1 (Reference) & & & \\
\hline Left & 0.986 (0.398-2.445) & 0.976 & & \\
\hline Bilateral & NA & NA & & \\
\hline \multicolumn{5}{|l|}{ BPH history } \\
\hline No & 1 (Reference) & & 1 (Reference) & \\
\hline Yes & $6.512(2.560-16.561)$ & $<0.001$ & $2.090(0.679-6.433)$ & 0.199 \\
\hline Operation time & 0.993(0.967-1.020) & 0.610 & $0.990(0.964-1.017)$ & 0.480 \\
\hline \multicolumn{5}{|l|}{ Postoperative pain NRS score } \\
\hline Immediate postoperative & 1.165 (0.815-1.666) & 0.403 & $1.130(0.777-1.644)$ & 0.541 \\
\hline Postoperative $6 \mathrm{hr}$ & 1.155 (0.882-1.513) & 0.295 & $1.110(0.827-1.490)$ & 0.509 \\
\hline Postoperative 24 hr & $1.196(0.820-1.744)$ & 0.354 & 1.147 (0.779-1.689) & 0.510 \\
\hline Wound seroma & $0.686(0.088-5.358)$ & 0.720 & $1.066(0.196-5.805)$ & 0.942 \\
\hline
\end{tabular}

$\mathrm{OR}$, odds ratio; $\mathrm{Cl}$, confidence interval; TEP, totally extraperitoneal repair; TAPP, transabdominal preperitoneal repair; BMl, body mass index; NA, not applicable; BPH, benign prostate hyperplasia; NRS, numeric rating scale. 


\section{Surgical outcomes}

The surgical outcomes of the two groups are presented in Table 2. The operation time was significantly shorter in the TEP group than in the TAPP group (43.1 \pm 14.9 minutes vs. $63.5 \pm 16.5$ min- utes, $p<0.001)$. Conversion to surgery occurred in seven patients and only in the TEP group, but the conversion rates did not differ significantly between the TEP and TAPP groups (3.1\% vs. $0 \%$, $p=0.053$ ). The mean postoperative pain scores were consistently lower in the TEP group than in the TAPP group. The difference

Table 4. Risk factor of chronic pain

\begin{tabular}{|c|c|c|c|c|}
\hline \multirow{2}{*}{ Variable } & \multicolumn{2}{|c|}{ Univariate } & \multicolumn{2}{|c|}{ Multivariate } \\
\hline & $\mathrm{OR}(95 \% \mathrm{Cl})$ & $p$ value & $\mathrm{OR}(95 \% \mathrm{Cl})$ & $p$ value \\
\hline \multicolumn{5}{|l|}{ Operation method } \\
\hline TEP & 1 (Reference) & & & \\
\hline TAPP & $1.939(0.385-9.76)$ & 0.422 & & \\
\hline Age & $0.987(0.942-1.034)$ & 0.589 & & \\
\hline \multicolumn{5}{|l|}{ Sex } \\
\hline Female & 1 (Reference) & & & \\
\hline Male & 1.193 (0.062-22.797) & 0.903 & 1 (Reference) & \\
\hline BMl & $0.699(0.494-0.988)$ & 0.043 & 0.708 (0.499-1.005) & 0.053 \\
\hline \multicolumn{5}{|l|}{ ASA PS classification } \\
\hline । & 1 (Reference) & & & \\
\hline$\|$ & $1.15(0.207-6.394)$ & 0.873 & & \\
\hline III & NA & NA & & \\
\hline IV & NA & NA & & \\
\hline \multicolumn{5}{|l|}{ Type } \\
\hline Direct & 1 (Reference) & & & \\
\hline Indirect & $0.412(0.082-2.08)$ & 0.283 & & \\
\hline Femoral & NA & NA & & \\
\hline Pantaloon & NA & NA & & \\
\hline \multicolumn{5}{|l|}{ Location } \\
\hline Right & 1 (Reference) & & & \\
\hline Left & 0.235 (0.027-2.032) & 0.188 & & \\
\hline Bilateral & NA & NA & & \\
\hline \multicolumn{5}{|l|}{ BPH history } \\
\hline No & 1 (Reference) & & 1 (Reference) & \\
\hline Yes & $5.76(1.131-29.346)$ & 0.035 & 5.363 (1.028-27.962) & 0.046 \\
\hline Operation time & $0.985(0.936-1.037)$ & 0.564 & $0.974(0.921-1.03)$ & 0.353 \\
\hline No. of Nelaton catheterizations & $2.395(0.987-5.811)$ & 0.053 & & \\
\hline \multicolumn{5}{|l|}{ Postoperative pain NRS score } \\
\hline Immediate postoperative & NA & NA & & \\
\hline Postoperative $6 \mathrm{hr}$ & 1.495 (0.783-2.855) & 0.223 & $1.548(0.718-3.335)$ & 0.265 \\
\hline Postoperative $24 \mathrm{hr}$ & $1.224(0.764-1.961)$ & 0.400 & $1.015(0.556-1.853)$ & 0.961 \\
\hline Wound seroma & $1.558(0.924-2.627)$ & 0.096 & $1.415(0.765-2.617)$ & 0.269 \\
\hline
\end{tabular}

OR, odds ratio; Cl, confidence interval; TEP, totally extraperitoneal repair; TAPP, transabdominal preperitoneal repair; BMl, body mass index; ASA, American Society of Anesthesiologists; PS, physical status; NA, not applicable; BPH, benign prostate hyperplasia; NRS, numeric rating scale. 
was statistically significant immediately ( $3.6 \pm 1.3$ vs. $4.4 \pm 1.1, p<$ $0.001)$, and 6 hours $(1.5 \pm 1.4$ vs. $2.3 \pm 1.8, p<0.001)$ after surgery. However, the difference was no longer significant 24 hours after surgery $(0.4 \pm 0.9$ vs. $0.6 \pm 1.2, p=0.070)$. The postoperative hospital stay was slightly shorter in the TEP group than in the TAPP group, but this difference was not significant ( $1.2 \pm 0.5$ vs. $1.3 \pm$ 0.6 days, $p=0.062$ ). The numbers of urinary catheterizations and Foley catheter insertion events did not differ significantly between groups. The overall complication rate was the same (11.9\%) in both groups; 27 of 226 cases in the TEP group and 14 of 118 cases in the TAPP group. The incidence rates of wound seroma, infection and hematoma, presence of chronic pain, mesh removal, and recurrence did not differ significantly between groups.

We tried to identify the risk factors for POUR, as represented by the need for postoperative Foley catheter insertion. In the univariate analysis, age and $\mathrm{BPH}$ history were significant risk factors for Foley catheter insertion, with odds ratios (ORs) of 1.116 ( $p<$ $0.001)$ and $6.512(p<0.001)$, respectively (Table 3$)$. After multivariate analysis, only age remained significant, with an OR of 1.083 ( $p=0.011$. Other demographics and surgical outcomes, including postoperative complications, were not significant risk factors for POUR.

We also tried to identify the risk factors related to chronic pain after laparoscopic inguinal hernia repair. In the univariate analysis, BMI and BPH history were significantly associated with chronic pain; ORs of $0.699(p=0.043)$ and $5.760(p=0.035)$, respectively (Table 4). After multivariate analysis, $\mathrm{BPH}$ history was the only risk factor related to the occurrence of chronic pain with an OR of $5.363(p=0.046)$.

\section{DISCUSSION}

Two methods of laparoscopic inguinal hernia repair are used at present; TEP and TAPP. In both procedures, mesh is inserted into the preperitoneal space, but the methods of access to reach this plane differ between TEP and TAPP. These two techniques have their advantages and disadvantages, mainly those relating directly to the method of access. In TEP, a totally preperitoneal approach is used with or without a dissecting balloon. This procedure makes it unnecessary to open and close the peritoneum, and allows exploration of the myopectineal orifice without the need to enter the abdominal cavity. This procedure has a shorter operation time and has a theoretical advantage for the postoperative recovery by preventing peritoneal irritation and adhesion. However, its narrow surgical field and unusual anatomical view require a longer learning curve compared with TAPP. In TAPP, the laparoscopic exploration makes the anatomy easier to identify and the presence of hernia on the contralateral side to be examined intuitively.

Studies have tried to determine which method is better. No significant difference was found for the postoperative recurrence rate between the two, and both are regarded as acceptable treatment options for inguinal hernia repair $[5,6]$. However, there are diverse opinions about the occurrence of perioperative complications. In this study, we compared the surgical outcomes of the two methods, such as the extent of postoperative acute and chronic pain, and POUR, and tried to identify risk factors for POUR and chronic pain, which are the representative complications of hernia repair in the early and late postoperative period.

The NRS pain scores up to 6 hours after operation were significantly higher in the TAPP group, but chronic pain did not differ significantly between the two groups. Bansal et al. [11] reported greater pain up to 6 weeks after TAPP than after TEP surgery, but little difference after that. However, Wei et al. [12] reported similar postoperative pain between the two methods. Although postoperative pain is influenced by the kinds of mesh and fixation modalities [13-16], we used the same type of mesh and fixation modalities for both techniques and were able to exclude the influence of the material. In our study, the operation time was slightly longer in the TAPP than in the TEP group, and it is likely that this reflected the time needed to open and close the peritoneum. This is similar to the results of another study [11].

We found no significant differences in postoperative complications, relapse, and chronic pain between the two methods, which are similar results to those of previous studies $[5,6]$. Taken together, these findings indicate that both methods are safe.

We found little difference between the two methods for the rates of urinary catheterization or need for Foley catheter insertion, which suggests that the method is not a risk factor of POUR. The only risk factor related to the incidence of POUR in the multivariate analysis was age. This is probably because the contractility and pressure of the detrusor muscle deteriorate progressively with advancing age $[17,18]$. Therefore, clinicians should pay more attention to POUR in aged patients.

In the current study, lower BMI and BPH history were related to chronic pain in the univariate analysis, but $\mathrm{BPH}$ history was the only significant risk factor after the multivariate analysis. Several studies reported that the extent of pain during the first week after surgery was associated with the rate of chronic pain $[19,20]$; however, in our study, the pain score during the early postoperative periods was not related to the incidence of chronic pain. Although a previous study reported that BPH history was not related to chronic pain [16], we found the opposite, and further studies are needed to clarify this relationship. The cause of chronic pain after a hernia surgery can be multifactorial and cannot be identified clearly, in many patients. Pain can be caused by neural damage or being trapped by a fixing device or a suture. A trapped nerve can also occur when the mesh becomes folded or wrinkles as it shrinks, a condition called meshoma [21]. It is reasonable to think that the shrinkage of a mesh, which is com- 
mon, can trap a nerve and cause chronic pain. Pain can also be caused by inflammation around the mesh. The abdominal pressure increases more during voiding in patients with $\mathrm{BPH}$ than in those without. We suspect that, after inguinal hernia surgery, increased abdominal pressure may affect those patients whose nerve is sutured by a fixation device or becomes trapped by mesh shrinkage, and possibly even in patients whose nerve is not damaged.

This study has some limitations. As a retrospective study, there may have been a selection bias in the study, but we found few differences in the patient characteristics between the selected groups. Ultrasound and computed tomography were not performed comprehensively on all the patients, and the exact defect sizes were not recorded in all operative notes, so the defect size was not included in the analyses. Given that the defect size is related to the rate of complications or reoperation [6], the lack of data about defect size may have affected the results of our study. However, the open technique is selected for patients with a large defect, which means that such patients were excluded from our study and, therefore, this may have minimized any effect of the defect size. Future studies should investigate the relationship between the increase in abdominal pressure caused by BPH and chronic pain by measuring intra-abdominal pressure in patients given urinary catheterization.

In conclusion, the operation time was shorter, and pain scores immediately and 6 hours after operation were lower for TEP than for TAPP; however, the other early and late complications did not differ between procedures. Both methods seem to be safe and effective for laparoscopic inguinal hernia repair. Age was a significant risk factor for POUR, and BPH history was a significant risk factor for chronic pain. For patients with these risk factors, careful observation of bladder function during hospitalization and proper outpatient follow-up are recommended.

\section{NOTES}

\section{Ethical statements}

This retrospective study was performed in accordance with the Declaration of Helsinki; this study was approved by the Institutional Review Board of Kangbuk Samsung Hospital (No. KBSMC 2021-11-007), and informed consent was waived.

\section{Authors' contributions}

Conceptualization: SGK, SRL, KUJ

Data curation: SGK, JS

Formal analysis: SGK

Investigation: SGK, JS

Methodology: SGK, KUJ
Project administration: SRL, KUJ

Visualization: SGK, KUJ

Writing-original draft: SGK, JS

Writing-review \& editing: SRL, KUJ

All authors read and approved the final manuscript.

\section{Conflict of interest}

All authors have no conflicts of interest to declare.

\section{Funding/support}

None.

\section{ORCID}

Sung Gu Kim, https://orcid.org/0000-0002-2331-1157 Jungtack Son, https://orcid.org/0000-0001-8898-2691

Sung Ryol Lee, https://orcid.org/0000-0001-9963-3673

Kyung Uk Jung, https://orcid.org/0000-0002-8844-3724

\section{REFERENCES}

1. Bradley M, Morgan D, Pentlow B, Roe A. The groin hernia: an ultrasound diagnosis? Ann R Coll Surg Engl 2003;85:178-180.

2. Fitzgibbons RJ Jr, Giobbie-Hurder A, Gibbs JO, et al. Watchful waiting vs repair of inguinal hernia in minimally symptomatic men: a randomized clinical trial. JAMA 2006;295:285-292.

3. Arregui ME, Davis CJ, Yucel O, Nagan RF. Laparoscopic mesh repair of inguinal hernia using a preperitoneal approach: a preliminary report. Surg Laparosc Endosc 1992;2:53-58.

4. McKernan JB, Laws HL. Laparoscopic repair of inguinal hernias using a totally extraperitoneal prosthetic approach. Surg Endosc 1993;7:26-28.

5. Bittner R, Montgomery MA, Arregui E, et al. Update of guidelines on laparoscopic (TAPP) and endoscopic (TEP) treatment of inguinal hernia (International Endohernia Society). Surg Endosc 2015;29:289321.

6. Köckerling F, Bittner R, Jacob DA, et al. TEP versus TAPP: comparison of the perioperative outcome in 17,587 patients with a primary unilateral inguinal hernia. Surg Endosc 2015;29:3750-3760.

7. HerniaSurge Group. International guidelines for groin hernia management. Hernia 2018;22:1-165.

8. Alfieri S, Amid PK, Campanelli G, et al. International guidelines for prevention and management of post-operative chronic pain following inguinal hernia surgery. Hernia 2011;15:239-249.

9. Andresen K, Rosenberg J. Management of chronic pain after hernia repair. J Pain Res 2018;11:675-681.

10. Werner MU, Kongsgaard UE. I. Defining persistent post-surgical pain: is an update required? $\mathrm{Br} J$ Anaesth 2014;113:1-4. 
11. Bansal VK, Misra MC, Babu D, et al. A prospective, randomized comparison of long-term outcomes: chronic groin pain and quality of life following totally extraperitoneal (TEP) and transabdominal preperitoneal (TAPP) laparoscopic inguinal hernia repair. Surg Endosc 2013;27:2373-2382.

12. Wei FX, Zhang YC, Han W, Zhang YL, Shao Y, Ni R. Transabdominal preperitoneal (TAPP) versus totally extraperitoneal (TEP) for laparoscopic hernia repair: a meta-analysis. Surg Laparosc Endosc Percutan Tech 2015;25:375-383.

13. Belyansky I, Tsirline VB, Klima DA, Walters AL, Lincourt AE, Heniford TB. Prospective, comparative study of postoperative quality of life in TEP, TAPP, and modified Lichtenstein repairs. Ann Surg 2011;254:709-715.

14. Brügger L, Bloesch M, Ipaktchi R, Kurmann A, Candinas D, Beldi G. Objective hypoesthesia and pain after transabdominal preperitoneal hernioplasty: a prospective, randomized study comparing tissue adhesive versus spiral tacks. Surg Endosc 2012;26:1079-1085.

15. Li W, Sun D, Sun Y, et al. The effect of transabdominal preperitoneal (TAPP) inguinal hernioplasty on chronic pain and quality of life of patients: mesh fixation versus non-fixation. Surg Endosc
2017;31:4238-4243.

16. Lo CW, Chen YT, Jaw FS, Yu CC, Tsai YC. Predictive factors of postlaparoscopic inguinal hernia acute and chronic pain: prospective follow-up of 807 patients from a single experienced surgeon. Surg Endosc 2021;35:148-158.

17. Pfisterer MH, Griffiths DJ, Schaefer W, Resnick NM. The effect of age on lower urinary tract function: a study in women. J Am Geriatr Soc 2006;54:405-412.

18. Cho KJ, Kim JC. Pathophysiology of lower urinary tract dysfunction in the older patient. J Korean Med Assoc 2015;58:868-872.

19. Singh AN, Bansal VK, Misra MC, et al. Testicular functions, chronic groin pain, and quality of life after laparoscopic and open mesh repair of inguinal hernia: a prospective randomized controlled trial. Surg Endosc 2012;26:1304-1317.

20. Tolver MA, Rosenberg J, Bisgaard T. Early pain after laparoscopic inguinal hernia repair: a qualitative systematic review. Acta Anaesthesiol Scand 2012;56:549-557.

21. Amid PK. Radiologic images of meshoma: a new phenomenon causing chronic pain after prosthetic repair of abdominal wall hernias. Arch Surg 2004;139:1297-1298. 